\title{
An Optimization model for Triple Sourcing, Supply Chain under Disruption
}

\author{
Hegazy Zaher \\ Department of Mathematical \\ Statistics \\ Institute of Statistical studied \& \\ Research \\ Cairo University
}

\author{
Naglaa Ragaa \\ Department of Operations \\ Research \\ Institute of Statistical studied \& \\ Research \\ Cairo University
}

\author{
Marwa Mostafa Selim \\ Department of Operations \\ Research \\ Institute of Statistical studied \& \\ Research \\ Cairo University
}

\begin{abstract}
The trade-off among ordering policies and disruption risks in sourcing networks is a critical issue. This research proposes a generic newsvendor type model for triple sourcing, supply chains where all of the three chains subjected to disrupt events. It is assumed that each supply channel is susceptible to network, operations, and external risks or a combination of all of them. Optimal solutions are obtained in the ordering quantities and the total expected profit is computed. An illustrative numerical example is performed to help managers in balancing the ordering strategies under supply chains' risks.
\end{abstract}

\section{Keywords}

Demand uncertainty, triple sourcing, supply chain risk management, stochastic model

\section{INTRODUCTION}

Studying supply chain risk management is becoming an increasingly important issue in recent years due to world catastrophic like a tsunami in Japan, SARS virus in 2003 and earthquake in Taiwan in 1999. Also, September 11 terrorist attack in 2001 raises risks of supply to global organizations that depends on outsourcing strategies. These external risk events and many others raised from the supply network itself make companies strive to manage supply chain risks. It is used to define supply risk as "the probability of suffering a loss" [1]. Galway [2] states that risk is an event, which is uncertain and has a negative impact on some endeavor. These definitions give a clear vision of risks to deal with them.

According to Hugo [3], organizations measure their performance and efficiency in supply chains by making decisions in these five areas; these are (Production, Inventory, Location, Transportation, and Information). However, vulnerability and sensitivity of supply networks towards risks are difficult to understand and manage [4]. In general, supply chain risk management process can be classified into four stages; risk identification, risk assessment, implementation of risk management actions and finally monitoring and controlling risks [5]. These risk management stages are most common in supply chain networks and these can help decision makers to face different risk consequences by themselves [6].

There exist many literatures related to sourcing strategies, risk management and decision-making under uncertainty. Here, recent research work is illustrated by reviewing single and dual sourcing methods in supply chains. To help managers in the decision making process of defining the reparable parts in the remanufacturing process that improve a responsive vendor with a reactive capacity, Ferrer [7] introduces optimal lotsize strategies. For a two-stage inventory and production control system, Xia et al. [8], provide a general manor and major risk management approach. Two problems are defined, with flexible setup time and with fixed setup time.

For a dual sourcing, supply chain, newsvendor models are introduced for both risk- averse and risk neutral decision makers where both channels subject to risks [9]. The double effects of supply chain visibility and supply chain risk on supply chain performance are explored by $\mathrm{Yu}$ et. al. [10]. They utilize a fuzzy multi-objective decision making approach to model Supply Chain Visibility and Supply Chain Risk from an operational perspective. Lately, Xia [11], develops a scenario of how a retailer could use its pricing and ordering decisions to respond to its supplier's temporary price discounts for one-retailer and one-supplier supply chain.

This paper is mainly focused on the disruption risks of a triple sourcing, supply chain with same procurement prices but different disruption probabilities and that can differentiate it from the existing literature. More specifically, generic stochastic newsvendor ordering model for risk neutral decision makers are proposed for a supply chain network of three unreliable suppliers and one retailer. The objective is to capture the trade-off among ordering policies and disruption risks in a triple-sourcing network, assuming that all suppliers are subjected to disruption risks.

The following sections of the paper are organized as follows. Section 2 defines the problem clearly then the proposed risk neutral decision making model is introduced in section 3. An illustrative numerical example is given to show the optimal ordering quantities that can be obtained from each supplier in section 4 and both of the total profit of the retailer and the behavior of decisions held by decision makers are discussed through the numerical results of this example in section 5 . Finally, a general conclusion is provided in section 6 .

\section{PROBLEM DEFINITION}

Here, it is assumed that the supply chain is for one retailer and three competing unreliable suppliers which are susceptible to several supply chain disruptions. The retailer must take a decision for the required quantities from each supply under demand uncertainty and before the sales period begins. Emergency replenishment ordering policy is not allowed.

Each supplier from the three suppliers is exposed to one type of risk event or for a combination of them. Risk events can be categorized according to Handfield and McCormack [12], into three types; (1) Network Risks that include five risk events (misalignment of interest, supplier financial stress, supplier leadership change, Tier 2 stoppage and supplier network misalignment), (2) Operational Risks that include four risk events (quality problems, delivery problems, supplier human resource problems and service problems), and (3) External 
Risks that include three risk events ( Supplier locked or shut down, merger/divestiture and disasters).

When a disruption occurs, it is assumed that the supply chain can provide only a percentage of the ordering quantity to the retailer (through the selling period). Different risk probabilities are considered for the three supply sources. The objective of the model is to maximize total profit after determining the appropriate quantity from each supplier.

\section{PROPOSED MODEL}

Demand $\mathrm{X}$ for the required product is assumed to be a positive stochastic random variable with probability density function $\mathrm{f}(\mathrm{x})$ and cumulative distribution function $\mathrm{F}(\mathrm{x})$. The selling period can be considered as $\mathrm{T}$ time unit while the selling price can be as $s . c_{i}$ is the unit cost paid to supplier $i$

$(i=1,2,3)$ where it is assumed that the selling is greater than the unit cost of each supplier $\left(s>c_{i}\right)$. At the end of the selling period, the remaining units can be sold to a secondary market or with a discount at a unit salvage value $r$ less than the unit cost $\left(r<c_{i}\right)$. Also, $k$ represents the cost associated with the unit shortage.

The probability of risk events for each supply chain $i$ is introduced by pi and it is assumed that only a percentage of the quantities from the three suppliers will reach the retailer shop space when disruptions occur and it can be expressed by $\mathrm{y}_{\mathrm{i}}$.

The following part of the research will introduce the base cases of unconstrained model for risk-neutral managers then a numerical analysis can be illustrated to help in decision processes.

The decision maker needs to reach the maximum profit under risk and so this profit can be obtained from maximizing the objective function as follows

The optimization model, $(P)$ to be maximized is

$$
(P)=\max G\left(Q_{1}, Q_{2}, Q_{3}\right)
$$

$G\left(Q_{1}, Q_{2}, Q_{3}\right)=\left(1-p_{1}\right)\left(1-p_{2}\right)\left(1-p_{3}\right) G_{0}\left(Q_{1}, Q_{2}, Q_{3}\right)+p_{1}\left(1-p_{2}\right)\left(1-p_{3}\right) G_{1}\left(Q_{1}, Q_{2}, Q_{3}\right)$ $+\left(1-p_{1}\right) p_{2}\left(1-p_{3}\right) G_{2}\left(Q_{1}, Q_{2}, Q_{3}\right)+\left(1-p_{1}\right)\left(1-p_{2}\right) p_{3} G_{3}\left(Q_{1}, Q_{2}, Q_{3}\right)$ $+p_{1} p_{2}\left(1-p_{3}\right) G_{12}\left(Q_{1}, Q_{2}, Q_{3}\right)+p_{1}\left(1-p_{2}\right) p_{3} G_{13}\left(Q_{1}, Q_{2}, Q_{3}\right)$ $+\left(1-p_{1}\right) p_{2} p_{3} G_{23}\left(Q_{1}, Q_{2}, Q_{3}\right)+p_{1} p_{2} p_{3} G_{123}\left(Q_{1}, Q_{2}, Q_{3}\right)$

When a disruption occurs on the supplier, the following cases can represent the collection of all possible combinations of risk events on none, on only one, on two or on all of the three supply channels.

The $1^{\text {st }}$ case: when none of the three suppliers' channel faces a disruption

When no risk factor or a combination of risk factors affects the three suppliers' channels, the classical newsvendor formula will be changed and the wholesaler expected gain can be calculated as follows (with Probability $=\left(1-p_{1}\right)\left(1-p_{2}\right)\left(1-p_{3}\right)$

and Quantity delivered $\left.=Q_{1}, Q_{2}, Q_{3}\right)$

$$
\begin{aligned}
G_{0}\left(Q_{1}, Q_{2}, Q_{3}\right)= & \int_{0}^{Q_{1}+Q_{2}+Q_{3}}\left[s x-c_{1} Q_{1}-c_{2} Q_{2}-c_{3} Q_{3}+r\left(Q_{1}+Q_{2}+Q_{3}-x\right)\right] f(x) d x \\
& +\int_{Q_{1}+Q_{2}+Q_{3}}^{\infty}\left[s\left(Q_{1}+Q_{2}+Q_{3}\right)-c_{1} Q_{1}-c_{2} Q_{2}-c_{3} Q_{3}-k\left(x-Q_{1}-Q_{2}-Q_{3}\right] f(x) d x\right.
\end{aligned}
$$

The $2^{\text {nd }}$ case: When a disruption occurs only on the first supplier's channel
The risk factor or a combination of risk factors affects only the first supplier's channel, the expected gain can be provided by (With Probability $=p_{1}\left(1-p_{2}\right)\left(1-p_{3}\right)$ and Quantity delivered $\left.=y_{1} Q_{1}, Q_{2}, Q_{3}\right)$

$$
\begin{aligned}
G_{1}\left(Q_{1}, Q_{2}, Q_{3}\right)= & \int_{0}^{y_{1} Q_{1}+Q_{2}+Q_{3}}\left[s x-y_{1} c_{1} Q_{1}-c_{2} Q_{2}-c_{3} Q_{3}+r\left(y_{1} Q_{1}+Q_{2}+Q_{3}-x\right)\right] f(x) d x \\
& +\int_{y_{1} Q_{1}+Q_{2}+Q_{3}}^{\infty}\left[s\left(y_{1} Q_{1}+Q_{2}+Q_{3}\right)-y_{1} c_{1} Q_{1}-c_{2} Q_{2}-c_{3} Q_{3}-k\left(x-y_{1} Q_{1}-Q_{2}-Q_{3}\right] f(x) d x\right.
\end{aligned}
$$

The $3^{\text {rd }}$ case: When a disruption occurs only in the second supplier's channel

The risk factor or a combination of risk factors affects only the second supplier's channel, the expected gain can be provided by (With Probability $=\left(1-p_{1}\right) p_{2}\left(1-p_{3}\right)$ and Quantity delivered $\left.=Q_{1}, y_{2} Q_{2}, Q_{3}\right)$

$$
\begin{aligned}
\boldsymbol{G}_{2}\left(\boldsymbol{Q}_{1}, \boldsymbol{Q}_{2}, \boldsymbol{Q}_{3}\right)= & \int_{0}^{Q_{1}+y_{2} Q_{2}+Q_{3}}\left[s x-c_{1} Q_{1}-y_{2} c_{2} Q_{2}-c_{3} Q_{3}+r\left(Q_{1}+y_{2} Q_{2}+Q_{3}-x\right)\right] f(x) d x \\
& +\int_{Q_{1}+y_{2} Q_{2}+Q_{3}}^{\infty}\left[s\left(Q_{1}+y_{2} Q_{2}+Q_{3}\right)-c_{1} Q_{1}-y_{2} c_{2} Q_{2}-c_{3} Q_{3}-k\left(x-Q_{1}-y_{2} Q_{2}-Q_{3}\right] f(x) d x\right.
\end{aligned}
$$

The $4^{\text {th }}$ case: When a disruption occurs only on the third supplier's channel

The risk factor or a combination of risk factors affects only the third supplier's channel, the expected gain can be provided by (With Probability $=\left(1-p_{1}\right)\left(1-p_{2}\right) p_{3}$ and Quantity delivered $\left.=Q_{1}, Q_{2}, y_{3} Q_{3}\right)$

$$
\begin{aligned}
\boldsymbol{G}_{3}\left(\boldsymbol{Q}_{1}, \boldsymbol{Q}_{2}, \boldsymbol{Q}_{3}\right)= & \int_{0}^{Q_{1}+Q_{2}+y_{3} Q_{3}}\left[s x-c_{1} Q_{1}-c_{2} Q_{2}-y_{3} c_{3} Q_{3}+r\left(Q_{1}+Q_{2}+y_{3} Q_{3}-x\right)\right] f(x) d x \\
& +\int_{Q_{1}+Q_{2}+y_{3} Q_{3}}^{\infty}\left[s\left(Q_{1}+Q_{2}+y_{3} Q_{3}\right)-c_{1} Q_{1}-c_{2} Q_{2}-y_{3} c_{3} Q_{3}-k\left(x-Q_{1}-Q_{2}-y_{3} Q_{3}\right] f(x) d x\right.
\end{aligned}
$$

The $5^{\text {th }}$ case: When a disruption occurs only on the first and the second suppliers' channels

The risk factor or a combination of risk factors affects the first and the second supplier's channels, the expected gain can be provided by (With Probability $=p_{1} p_{2}\left(1-p_{3}\right)$ and Quantity

$$
\begin{aligned}
& \text { delivered } \left.=y_{1} Q_{1}, y_{2} Q_{2}, Q_{3}\right) \\
& \begin{aligned}
G_{12}\left(Q_{1}, Q_{2}, Q_{3}\right) & =\int_{0}^{y_{1} Q_{1}+y_{2} Q_{2}+Q_{3}}\left[s x-y_{1} c_{1} Q_{1}-y_{2} c_{2} Q_{2}-c_{3} Q_{3}+r\left(y_{1} Q_{1}+y_{2} Q_{2}+Q_{3}-x\right)\right] f(x) d x \\
& +\int_{y_{1} Q_{1}+y_{2} Q_{2}+Q_{3}}^{\infty}\left[s\left(y_{1} Q_{1}+y_{2} Q_{2}+Q_{3}\right)-y_{1} c_{1} Q_{1}-y_{2} c_{2} Q_{2}-c_{3} Q_{3}-k\left(x-y_{1} Q_{1}-y_{2} Q_{2}-Q_{3}\right] f(x) d x\right.
\end{aligned}
\end{aligned}
$$

The $6^{\text {th }}$ case: when a disruption occurs only on the first and the third suppliers' channels

The risk factor or a combination of risk factors affects the first and the third supplier's channel (With Probability = $p_{1}\left(1-p_{2}\right) p_{3}$ and Quantity delivered $y_{1} Q_{1}, Q_{2}, y_{3} Q_{3}$

$$
\begin{aligned}
\boldsymbol{G}_{13}\left(\boldsymbol{Q}_{1}, Q_{2}, Q_{3}\right) & =\int_{0}^{y_{1} Q_{1}+Q_{2}+y_{3} Q_{3}}\left[s x-y_{1} c_{1} Q_{1}-c_{2} Q_{2}-y_{3} c_{3} Q_{3}+r\left(y_{1} Q_{1}+Q_{2}+y_{3} Q_{3}-x\right)\right] f(x) d x \\
& +\int_{y_{1} Q_{1}+Q_{2}+y_{3} Q_{3}}^{\infty}\left[s\left(y_{1} Q_{1}+Q_{2}+y_{3} Q_{3}\right)-y_{1} c_{1} Q_{1}-c_{2} Q_{2}-y_{3} c_{3} Q_{3}-k\left(x-y_{1} Q_{1}-Q_{2}-y_{3} Q_{3}\right] f(x) d x\right.
\end{aligned}
$$

The $7^{\text {th }}$ case: when a disruption occurs only on the second and the third suppliers' channels

The risk factor or a combination of risk factors affects the second and the third suppliers' channel (With Probability = $\left(1-p_{1}\right) p_{2} p_{3}$ and Quantity delivered $\left.=Q_{1}, y_{2} Q_{2}, y_{3} Q_{3}\right)$

$$
\begin{aligned}
G_{23}\left(Q_{1}, Q_{2}, Q_{3}\right) & =\int_{0}^{Q_{1}+y_{2} Q_{2}+y_{3} Q_{3}}\left[s x-c_{1} Q_{1}-y_{2} c_{2} Q_{2}-y_{3} c_{3} Q_{3}+r\left(Q_{1}+y_{2} Q_{2}+y_{3} Q_{3}-x\right)\right] f(x) d x \\
& +\int_{Q_{1}+y_{2} Q_{2}+y_{3} Q_{3}}^{\infty}\left[s\left(Q_{1}+y_{2} Q_{2}+y_{3} Q_{3}\right)-c_{1} Q_{1}-y_{2} c_{2} Q_{2}-y_{3} c_{3} Q_{3}-k\left(x-Q_{1}-y_{2} Q_{2}-y_{3} Q_{3}\right] f(x) d x\right.
\end{aligned}
$$

The $8^{\text {th }}$ case: when a disruption occurs at all suppliers' channels 
The risk factor or a combination of risk factors affects the three suppliers' channel (With Probability $=p_{1} p_{2} p_{3}$ and Quantity delivered $=y_{1} Q_{1}, y_{2} Q_{2}, y_{3} Q_{3}$ )

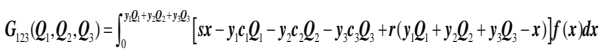

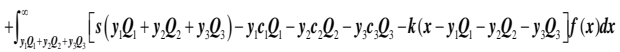

After illustrating the objective function (1) and the above cases (from equation 2 to equation 9) by collecting all possible combinations of risk events (on none, on only one, on two or on all of the three supply channels), the maximum profit can be calculated after differentiation of equations with respect to each supplier $\left(\mathrm{Q}_{1}, \mathrm{Q}_{2}\right.$, and $\left.\mathrm{Q}_{3}\right)$ then substitute these values in the objective function to get the optimal ordering quantities $\left(\mathrm{Q}_{1}{ }^{*}, \mathrm{Q}_{2}{ }^{*}, \mathrm{Q}_{3}{ }^{*}\right)$ from each supply chain then by solving the following system of equations (10), (11), and (12)

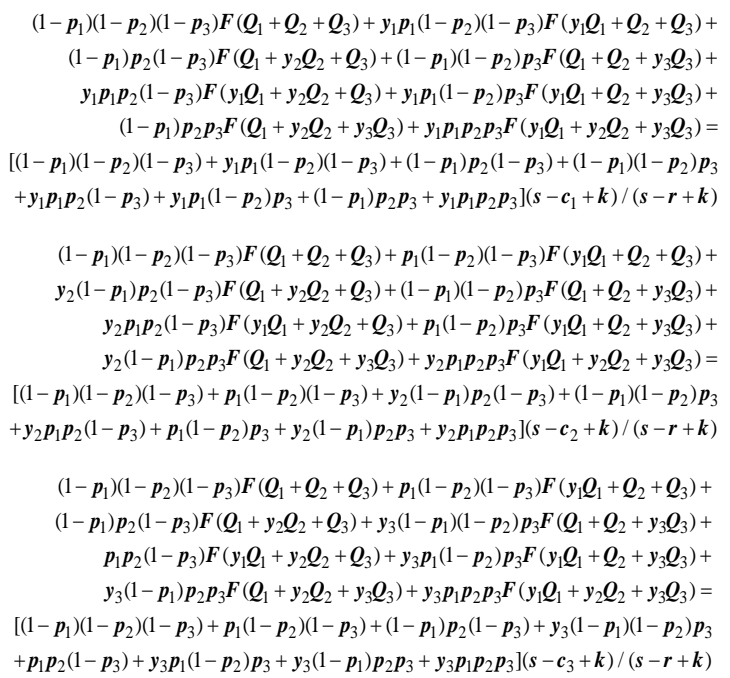

By assigning these optimal ordering values in the objective function the total maximum profit $G\left(Q_{1}, Q_{2}\right.$, and $\left.Q_{3}\right)$, can be determined.

\section{NUMERICAL EXAMPLE}

This research employs the risk events' survey and analysis made by Lockamy [13]. His study was carried out on 15 automotive casting suppliers and the first three suppliers are concerned in these numerical insights to understand the behavior of the introduced model.

The following table illustrates the probabilities of risk event $\left(p_{i} ; i=1,2,3\right)$.

"Table 1": The probabilities of risk events

\begin{tabular}{|c|c|c|c|c|}
\hline 离 & 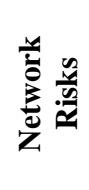 & 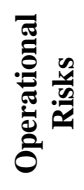 & 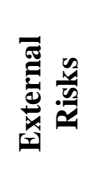 & 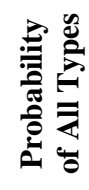 \\
\hline 1 & 0.34 & 0.47 & 0.43 & 0.41 \\
\hline 2 & 0.19 & 0.23 & 0.38 & 0.27 \\
\hline 3 & 0.33 & 0.46 & 0.43 & 0.41 \\
\hline
\end{tabular}

The values of unit selling price (s), salvage value (r) and lost sales' cost (k) is 45,10 , and 15 respectively. The unit supply cost paid to the first supplier is equal to 20 , while for the second supplier is equal to 21 and for the third is equal to 22 .
The percentage of the delivered quantity from each supplier when the risk occurs $y_{i}(i=1,2,3)$ is assumed to be 0.5 .

The retailer/wholesaler faces a demand uncertainty that follows the normal distribution, with a mean value equal to 400 units and standard deviation equals to 130 . Using the above parameter values, the model is solved to find the optimal solutions by Matlab7 (R2015a) software.

The results will be discussed in the following section.

\section{RESULTS}

When the probability of risk for the first supplier is equal to 0.34 and for the second supplier is equal to 0.19 and for the third supplier ranges by $0.33,0.46,0.43$, and 0.41 respectively, it can be estimated that the effect of risk events appears heavily on the third supplier (see figure 1), so the decision maker can order the largest quantity from the first then from the second supplier. The total profit decreases slightly with the increase of the probability of risk on the third supplier. The same results were observed with altering the probabilities of the three suppliers.

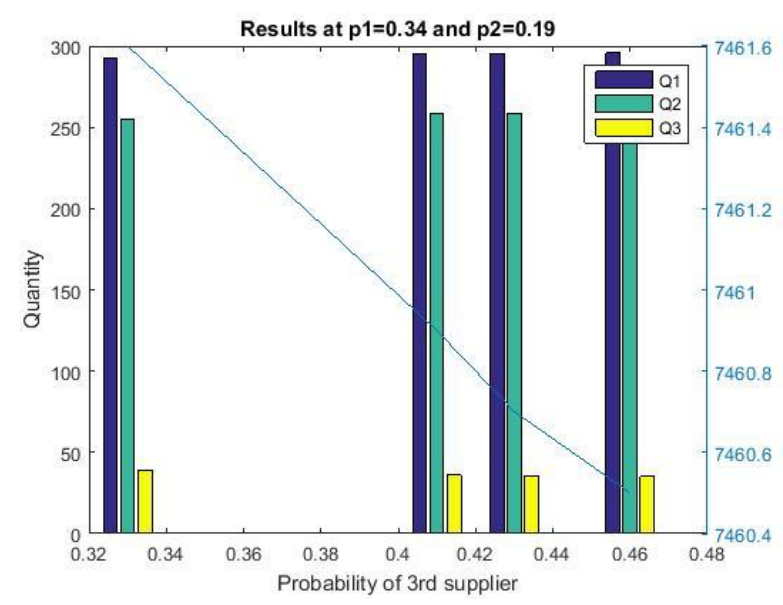

"Figure 1" The probability of the $3^{\text {rd }}$ supplier with the ordered quantity and the profit

Figure 2 shows the ranges of the ordered quantities from each supplier and the total profit. The maximum profit can be obtained when the probability of risk for the first, second and third suppliers are $0.34,0.19$, and 0.33 respectively.

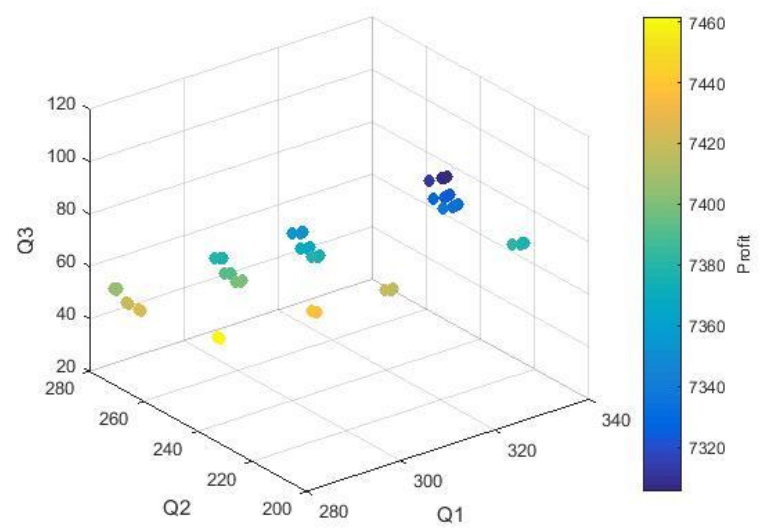

"Figure 2" The ordered quantities with the profit

For more results see Appendix 1. 


\section{CONCLUSION}

The great consequences of disruption events on supply chain networks make researchers give high attention to supply chain risk management. Different risk types can affect the sourcing process like Network, operational, external or a combination of them and that forces retailer to duplicate the number of suppliers. The probability of risk for each supplier can be estimated by several methods. The proposed model is a generic newsvendor type that can help risk neutral decision makers to manage both the consequences of risk events and the inventory policies by using triple sourcing strategies. The model gives the decision maker a clear vision on the optimal ordering quantities that can be ordered from each supplier and the expected total profit under disruption. A numerical example is given to illustrate the trend of the model towards different risk types. This decision making methodology can be used for several supply chains like remanufacturing and reverse channels not only the forward ones. Future works could be expanded to multi products or multi period for the supply chains. Also, the stochastic risk events and its consequences can be studied.

\section{REFERENCES}

[1] Mazareanu, V. 2007. Risk management and analysis: risk assessment (qualitative and quantitative).

[2] Galway, L. 2004. Quantitative risk analysis for project management: a critical review.

[3] Hugo, A. and Pistikopoulos, E.N. 2003. Environmentally conscious planning and design of supply chain networks.

[4] Waters, D. 2011. Supply chain risk management: vulnerability and resilience in logistics.
[5] Ritchie, B. and Brindley, C. 2007. Supply chain risk management and performance - A guiding framework for future development.

[6] Tuncel, G. and Alpan, G. 2010. Risk assessment and management for supply chain networks: a case study.

[7] Ferrer, G. 2003. Yield information and supplier responsiveness in remanufacturing operations.

[8] Xia, Y., Yang, M., Golany, B., Gilbert, S. and Yu, G. 2004. Real-time disruption management in a two-stage production and inventory system.

[9] Xanthopoulos, A., Vlachos, D. and Iakovou, E. 2012. Optimal newsvendor policies for dual-sourcing supply chains: A disruption risk management framework

[10] Yu, M. and Goh, M. 2014. A multi-objective approach to supply chain visibility and risk.

[11] Xia, Y. 2015. Responding to supplier temporary price discounts in a supply chain through ordering and pricing decisions.

[12] Handfield, R. and McCormack, K. 2007. Supply chain risk management: minimizing disruptions in global sourcing.

[13] Lockamy III, A. 2014. Assessing disaster risks in supply chains.

\section{APPENDIX}

"Table 1" Results when the probability of risk for the first supplier is equal to 0.34

\begin{tabular}{|c|c|c|c|c|c|c|c|}
\hline \multirow{2}{*}{ Solution No. } & $\mathbf{P}_{\mathbf{1}}$ & $\mathbf{P}_{\mathbf{2}}$ & $\mathbf{P}_{\mathbf{3}}$ & $\mathbf{Q}_{\mathbf{1}}$ & $\mathbf{Q}_{\mathbf{2}}$ & $\mathbf{Q}_{\mathbf{3}}$ & $\mathbf{G}$ \\
& & & & & & & \\
\hline $\mathbf{1}$ & 0.34 & 0.19 & 0.33 & 292.98 & 255.26 & 39.33 & 7461.61 \\
\hline $\mathbf{2}$ & 0.34 & 0.19 & 0.46 & 295.91 & 259.26 & 35.03 & 7460.54 \\
\hline $\mathbf{3}$ & 0.34 & 0.19 & 0.43 & 295.39 & 258.56 & 35.67 & 7460.73 \\
\hline $\mathbf{4}$ & 0.34 & 0.19 & 0.41 & 295 & 258.02 & 36.21 & 7460.87 \\
\hline $\mathbf{5}$ & 0.34 & 0.23 & 0.33 & 303.7 & 239.08 & 52.39 & 7437.36 \\
\hline $\mathbf{6}$ & 0.34 & 0.23 & 0.46 & 307.75 & 244.07 & 47.02 & 7435.46 \\
\hline $\mathbf{7}$ & 0.34 & 0.23 & 0.43 & 307.04 & 243.19 & 47.82 & 7435.79 \\
\hline $\mathbf{8}$ & 0.34 & 0.23 & 0.41 & 306.5 & 242.52 & 48.49 & 7436.05 \\
\hline $\mathbf{9}$ & 0.34 & 0.38 & 0.33 & 327.54 & 206.85 & 82.37 & 7381.18 \\
\hline $\mathbf{1 0}$ & 0.34 & 0.38 & 0.46 & 334.33 & 213.84 & 75.2 & 7376.52 \\
\hline $\mathbf{1 1}$ & 0.34 & 0.38 & 0.43 & 333.13 & 212.6 & 76.22 & 7377.35 \\
\hline $\mathbf{1 2}$ & 0.34 & 0.38 & 0.41 & 332.22 & 211.66 & 77.1 & 7377.98 \\
\hline $\mathbf{1 3}$ & 0.34 & 0.27 & 0.33 & 312.09 & 226.87 & 62.77 & 7417.98 \\
\hline $\mathbf{1 4}$ & 0.34 & 0.27 & 0.46 & 317.07 & 232.58 & 56.68 & 7415.26 \\
\hline $\mathbf{1 5}$ & 0.34 & 0.27 & 0.43 & 316.19 & 231.57 & 57.57 & 7415.74 \\
\hline $\mathbf{1 6}$ & 0.34 & 0.27 & 0.41 & 315.52 & 230.81 & 58.32 & 7416.11 \\
\hline
\end{tabular}


“Table 2" Results when the probability of risk for the first supplier is equal to 0.41

\begin{tabular}{|c|c|c|c|c|c|c|c|}
\hline Solution No. & $\mathbf{P}_{1}$ & $\mathbf{P}_{2}$ & $\mathbf{P}_{3}$ & $\mathbf{Q}_{1}$ & $\mathbf{Q}_{2}$ & $\mathbf{Q}_{3}$ & G \\
\hline 1 & 0.41 & 0.19 & 0.33 & 282.77 & 266.79 & 49.28 & 7427.17 \\
\hline 2 & 0.41 & 0.19 & 0.46 & 286.41 & 271.85 & 44.03 & 7425.48 \\
\hline 3 & 0.41 & 0.19 & 0.43 & 285.77 & 270.96 & 44.81 & 7425.77 \\
\hline 4 & 0.41 & 0.19 & 0.41 & 285.28 & 270.29 & 45.47 & 7426 \\
\hline 5 & 0.41 & 0.23 & 0.33 & 293.95 & 250.18 & 63.28 & 7400.35 \\
\hline 6 & 0.41 & 0.23 & 0.46 & 298.82 & 256.28 & 57.01 & 7397.56 \\
\hline 7 & 0.41 & 0.23 & 0.43 & 297.96 & 255.2 & 57.93 & 7398.06 \\
\hline 8 & 0.41 & 0.23 & 0.41 & 297.31 & 254.38 & 58.7 & 7398.43 \\
\hline 9 & 0.41 & 0.38 & 0.33 & 318.84 & 217.12 & 95.54 & 7338 \\
\hline 10 & 0.41 & 0.38 & 0.46 & 326.71 & 225.3 & 87.64 & 7331.7 \\
\hline 11 & 0.41 & 0.38 & 0.43 & 325.32 & 223.84 & 88.75 & 7332.82 \\
\hline 12 & 0.41 & 0.38 & 0.41 & 324.26 & 222.74 & 89.71 & 7333.67 \\
\hline 13 & 0.41 & 0.27 & 0.33 & 302.7 & 237.64 & 74.42 & 7378.88 \\
\hline 14 & 0.41 & 0.27 & 0.46 & 308.59 & 244.48 & 67.47 & 7375.04 \\
\hline 15 & 0.41 & 0.27 & 0.43 & 307.55 & 243.27 & 68.48 & 7375.72 \\
\hline 16 & 0.41 & 0.27 & 0.41 & 306.76 & 242.35 & 69.34 & 7376.24 \\
\hline
\end{tabular}

"Table 3" Results when the probability of risk for the first supplier is equal to 0.43

\begin{tabular}{|c|c|c|c|c|c|c|c|}
\hline Solution No. & $\mathbf{P}_{1}$ & $\mathbf{P}_{2}$ & $\mathbf{P}_{3}$ & $\mathbf{Q}_{1}$ & $\mathbf{Q}_{2}$ & $\mathbf{Q}_{3}$ & G \\
\hline 1 & 0.43 & 0.19 & 0.33 & 281.51 & 269.23 & 51.36 & 7419.61 \\
\hline 2 & 0.43 & 0.19 & 0.46 & 285.33 & 274.51 & 45.92 & 7417.77 \\
\hline 3 & 0.43 & 0.19 & 0.43 & 284.65 & 273.58 & 46.73 & 7418.09 \\
\hline 4 & 0.43 & 0.19 & 0.41 & 284.14 & 272.87 & 47.41 & 7418.34 \\
\hline 5 & 0.43 & 0.23 & 0.33 & 292.85 & 252.5 & 65.56 & 7392.22 \\
\hline 6 & 0.43 & 0.23 & 0.46 & 297.93 & 258.83 & 59.1 & 7389.23 \\
\hline 7 & 0.43 & 0.23 & 0.43 & 297.04 & 257.71 & 60.05 & 7389.76 \\
\hline 8 & 0.43 & 0.23 & 0.41 & 296.36 & 256.87 & 60.85 & 7390.16 \\
\hline 9 & 0.43 & 0.38 & 0.33 & 318.15 & 219.22 & 98.31 & 7328.51 \\
\hline 10 & 0.43 & 0.38 & 0.46 & 326.3 & 227.64 & 90.26 & 7321.84 \\
\hline 11 & 0.43 & 0.38 & 0.43 & 324.85 & 226.14 & 91.38 & 7323.03 \\
\hline 12 & 0.43 & 0.38 & 0.41 & 323.76 & 225.01 & 92.36 & 7323.92 \\
\hline 13 & 0.43 & 0.27 & 0.33 & 301.74 & 239.88 & 76.87 & 7370.29 \\
\hline 14 & 0.43 & 0.27 & 0.46 & 307.86 & 246.95 & 69.75 & 7366.19 \\
\hline 15 & 0.43 & 0.27 & 0.43 & 306.78 & 245.7 & 70.77 & 7366.92 \\
\hline 16 & 0.43 & 0.27 & 0.41 & 305.96 & 244.76 & 71.65 & 7367.47 \\
\hline
\end{tabular}


"Table 4" Results when the probability of risk for the first supplier is equal to 0.47

\begin{tabular}{|c|c|c|c|c|c|c|c|}
\hline Solution No. & $\mathbf{P}_{1}$ & $\mathbf{P}_{2}$ & $\mathbf{P}_{3}$ & $\mathbf{Q}_{1}$ & $\mathbf{Q}_{2}$ & $\mathbf{Q}_{3}$ & G \\
\hline 1 & 0.47 & 0.19 & 0.33 & 281.11 & 273.08 & 54.65 & 7407.13 \\
\hline 2 & 0.47 & 0.19 & 0.46 & 285.25 & 278.71 & 48.9 & 7405.04 \\
\hline 3 & 0.47 & 0.19 & 0.43 & 284.52 & 277.72 & 49.75 & 7405.41 \\
\hline 4 & 0.47 & 0.19 & 0.41 & 283.97 & 276.97 & 50.47 & 7405.69 \\
\hline 5 & 0.47 & 0.23 & 0.33 & 292.86 & 256.16 & 69.16 & 7378.82 \\
\hline 6 & 0.47 & 0.23 & 0.46 & 298.33 & 262.84 & 62.4 & 7375.48 \\
\hline 7 & 0.47 & 0.23 & 0.43 & 297.36 & 261.66 & 63.39 & 7376.07 \\
\hline 8 & 0.47 & 0.23 & 0.41 & 296.63 & 260.76 & 64.22 & 7376.52 \\
\hline 9 & 0.47 & 0.38 & 0.33 & 319.11 & 222.43 & 102.7 & 7312.9 \\
\hline 10 & 0.47 & 0.38 & 0.46 & 327.83 & 231.21 & 94.36 & 7305.6 \\
\hline 11 & 0.47 & 0.38 & 0.43 & 326.29 & 229.65 & 95.51 & 7306.9 \\
\hline 12 & 0.47 & 0.38 & 0.41 & 325.11 & 228.47 & 96.52 & 7307.88 \\
\hline 13 & 0.47 & 0.27 & 0.33 & 302.07 & 243.38 & 80.72 & 7356.14 \\
\hline 14 & 0.47 & 0.27 & 0.46 & 308.64 & 250.8 & 73.31 & 7351.61 \\
\hline 15 & 0.47 & 0.27 & 0.43 & 307.48 & 249.49 & 74.38 & 7352.41 \\
\hline 16 & 0.47 & 0.27 & 0.41 & 306.6 & 248.49 & 75.29 & 7353.02 \\
\hline
\end{tabular}

